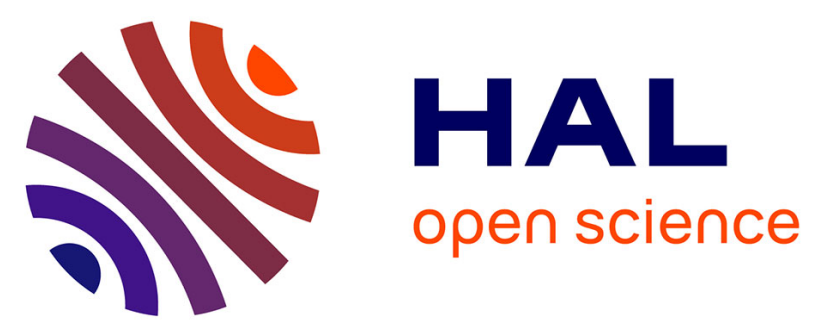

\title{
EEG-NIRS based assessment of neurovascular coupling during anodal transcranial direct current stimulation - a stroke case series
}

\author{
Anirban Dutta, Athira Jacob, Shubhajit Roy Chowdhury, Abhijit Das, \\ Michael Nitsche
}

\section{To cite this version:}

Anirban Dutta, Athira Jacob, Shubhajit Roy Chowdhury, Abhijit Das, Michael Nitsche. EEG-NIRS based assessment of neurovascular coupling during anodal transcranial direct current stimulation - a stroke case series. Journal of Medical Systems, 2015, 39 (4), pp.205-226. 10.1007/s10916-015-0205-7 . hal-01100750

\author{
HAL Id: hal-01100750 \\ https://hal.inria.fr/hal-01100750
}

Submitted on 16 Apr 2019

HAL is a multi-disciplinary open access archive for the deposit and dissemination of scientific research documents, whether they are published or not. The documents may come from teaching and research institutions in France or abroad, or from public or private research centers.
L'archive ouverte pluridisciplinaire HAL, est destinée au dépôt et à la diffusion de documents scientifiques de niveau recherche, publiés ou non, émanant des établissements d'enseignement et de recherche français ou étrangers, des laboratoires publics ou privés. 
Title: EEG-NIRS based assessment of neurovascular coupling during anodal transcranial direct current stimulation - a stroke case series

\section{Anirban Dutta, Athira Jacob, Shubhajit Roy Chowdhury, Abhijit Das, Michael A. Nitsche}

Correspondence: Dr. Anirban Dutta, is with the Charité - Universitätsmedizin Berlin, Germany and DEMAR (INRIA Sophia Antipolis), INRIA - CNRS : UMR5506 - Université Montpellier II - Sciences et techniques - Université Montpellier I, Montpellier, France, email:

anirban.dutta@charite.de

Ms. Athira Jacob is with the Indian Institute of Technology, Chennai, India.

Dr. S. Roy Chowdhury is with the International Institute of Information Technology,

Hyderabad, India.

Dr. med. A. Das is with the Institute of Neuroscience, Kolkata, India.

Dr. med. M. A. Nitsche is with the University of Medicine Goettingen, Germany.

\section{ABSTRACT}

Objective: A method for electroencephalography (EEG) - near-infrared spectroscopy (NIRS) based assessment of neurovascular coupling (NVC) during anodal transcranial direct current stimulation (tDCS).

Methods: Anodal tDCS modulates cortical neural activity leading to a hemodynamic response, which was used to identify impaired NVC functionality. In this study, the hemodynamic response was estimated with NIRS. NIRS recorded changes in oxy-hemoglobin ( $\mathrm{HbO2}$ ) and deoxyhemoglobin $(\mathrm{Hb})$ concentrations during anodal tDCS-induced activation of the cortical region located under the electrode and in-between the light sources and detectors. Anodal tDCS-induced alterations in the underlying neuronal current generators were also captured with EEG. Then, a method for the assessment of NVC underlying the site of anodal tDCS was proposed that leverages the Hilbert-Huang Transform.

Results: The case series including four chronic ( $>6$ months) ischemic stroke survivors (3 males, 1 female from age 31 to 76) showed non-stationary effects of anodal tDCS on EEG that correlated with the $\mathrm{HbO} 2$ response. Here, the initial dip in $\mathrm{HbO} 2$ at the beginning of anodal tDCS corresponded with an increase in the log-transformed mean-power of EEG within $0.5 \mathrm{~Hz}$ $11.25 \mathrm{~Hz}$ frequency band. The cross-correlation coefficient changed signs but was comparable across subjects during and after anodal tDCS. The log-transformed mean-power of EEG lagged $\mathrm{HbO} 2$ response during tDCS but then led post-tDCS.

Conclusion: This case series demonstrated changes in the degree of neurovascular coupling to a $0.526 \mathrm{~A} / \mathrm{m}^{2}$ square-pulse $(0-30 \mathrm{sec})$ of anodal tDCS. The initial dip in $\mathrm{HbO2}$ needs to be carefully investigated in a larger cohort, for example in patients with small vessel disease.

Keywords: neurovascular coupling, electroencephalography, near-infrared spectroscopy, Hilbert-Huang Transform. 


\section{INTRODUCTION}

Transcranial direct current stimulation (tDCS) has been shown to modulate cortical neural activity [1]. During such modulation, the electric currents from excitable membranes of brain tissue superimpose at a given location in the extracellular medium and generate a potential, which is referred to as the electroencephalogram (EEG) when recorded from the scalp [2]. Respective neural activity has been shown to be closely related, spatially and temporally, to cerebral blood flow (CBF) that supplies glucose via neurovascular coupling [3]. The hemodynamic response to neural activity can be captured by near-infrared spectroscopy (NIRS), which enables continuous monitoring of cerebral oxygenation and blood volume [4].

In our prior work, we have suggested that anodal tDCS enhances activity and excitability of the excitatory pyramidal neuron at a population level in a non-specific manner, and that $\mu$-rhythm desynchronization is generated [5]. The excitation versus inhibition effects of acute tDCS on the population kinetics can produce a whole spectrum of EEG signals within the oscillatory regime of a neural mass model [6]. The effects on the population kinetics depends on the direction of cortical current flow determining the relative influence of acute tDCS on the cellular targets responsible for the modulation of synaptic efficacy, which are concurrently somata and axon terminals [7]. Therefore, not all neural tissue will be equally affected by a given stimulation protocol which may distinctly affect neuronal populations/neuronal compartments. Here, basal and apical dendrites can be concomitantly polarized in opposite directions, and Layer $\mathrm{V}$ pyramidal neurons exhibit the highest measured somatic sensitivities to subthreshold fields [7]. Therefore, somatic depolarization of Layer V pyramidal neurons with anodal tDCS may result in

corresponding change in spontaneous firing rate [8] thereby causing rapid increase in extracellular potassium concentration due to outwardly directed potassium currents [9]. 
Moreover, Fricke and coworkers [10] hypothesized the role of L-type voltage-gated Ca2+ channels (L-VGCC) in short-term homeostatic plasticity since tDCS has been shown to induce a long-lasting disturbance of $\mathrm{Ca} 2+$ homeostasis [11] and induce calcium-dependent plasticity [12]. Here, the influence of long-lasting disturbance of $\mathrm{Ca} 2+$ homeostasis on the myogenic and the metabolic control of cerebral circulation cannot be excluded. The glial network has an important role in regulating neural activity by distributing extracellular ions, i.e. spatial buffering, which can have a time course of seconds [13]. Due to this relatively long time course, some of these diffusing extracellular potassium and calcium ions can act as mediators of vasodilation [14] as well as can act as neurotransmitters, affecting other neuronal compartments including GABAergic and glutamatergic synapses as a homeostatic feedback mechanism. Response to this homeostatic feedback might influence the aftereffects of anodal tDCS which have been shown to depend on the modulation of both GABAergic and glutamatergic synapses [15]. For example, depolarization of presynaptic glutamate terminals can increase the probability of glutamate release, and glutamate uptake into astrocytes may stimulate aerobic glycolysis - a mechanism coupling neuronal activity to glucose utilization [16]. Moreover, the action of GABA is primarily determined by the concentration of intracellular chloride. Prior work has shown that both, increasing and decreasing the extracellular potassium ion concentration results in a concomitant change in intracellular chloride concentration via potassium-coupled chloride cotransport [17].

Due to vessel dilation, the rates of glucose flux transfer across the endothelium into the interstitium increases. Consequently, astrocytes and neurons might uptake glucose (and lactate) from the interstitium to fuel recovery/maintenance of homeostasis during/following tDCS [18]. In accordance, our case series [19] showed detectable hemodynamic responses $(0-60 \mathrm{sec})$ to $0.526 \mathrm{~A} / \mathrm{m}^{2}$ square-pulse $(0-30 \mathrm{sec})$ of anodal $\mathrm{tDCS}$. It was postulated that alterations of the 
vascular system may result in delayed secondary changes of the cortical excitability. Indeed, recent work showed that lactate can modulate the activity of primary cortical neurons through a receptor-mediated pathway [20] and vasomotion rhythms can influence neural firing patterns [21].

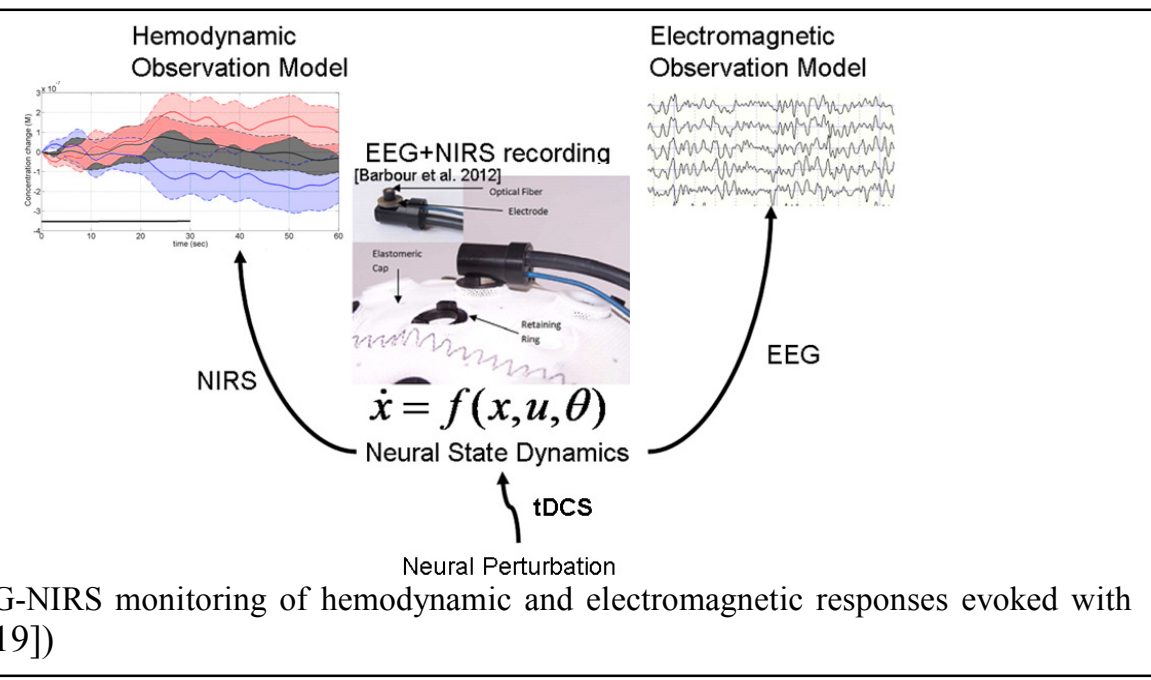

Based on these preliminary studies, we recently proposed EEG-NIRS-based screening and monitoring of neurovascular coupling functionality under perturbation with tDCS [22]. The objective of this methodological study is to present a neurovascular coupling model, and to assess its feasibility based on simultaneous EEG-NIRS recordings (simultaneous recording technique presented in Barbour et al. [23]) during anodal tDCS, as illustrated in Figure 1.

\section{METHODS}

\section{A. Case series}

Simultaneous recording of NIRS and EEG [23] during anodal tDCS was conducted on four chronic ( $>6$ months, see Table 1) ischemic stroke survivors after obtaining informed consent. All 
experiments were conducted in accordance with the Declaration of Helsinki at the Neuro Rehab Services LLP, India.

Table 1: Summary of the case series (M: male, F: female, MCA: middle cerebral artery, CABG: coronary artery bypass graft).

\begin{tabular}{|c|c|c|c|c|}
\hline Case & Age/gender & MRI diagnosis & Comorbidities & Year of stroke \\
\hline 1 & $31 / \mathrm{M}$ & Right MCA stroke & None & 2008 \\
\hline 2 & $63 / \mathrm{M}$ & Left MCA stroke & Diabetis, Hypertension & 2009 \\
\hline 3 & $73 / \mathrm{M}$ & Left MCA stroke & Post-CABG & 2010 \\
\hline 4 & $76 / \mathrm{F}$ & Right MCA stroke & Post-CABG & 2009 \\
\hline
\end{tabular}

B. Experimental protocol

Anodal tDCS (StarStim, Neuroelectrics, Spain) with the anode (SPONSTIM-8, Neuroelectrics, Spain) placed at $\mathrm{Cz}$ (international 10-20 system of scalp sites) and the cathode (SPONSTIM-25, Neuroelectrics, Spain) over left supraorbital notch was conducted with a current density of $0.526 \mathrm{~A} / \mathrm{m}^{2}$. tDCS was turned ON for $30 \mathrm{sec}$ and then OFF for $30 \mathrm{sec}$, which was repeated 15 times. Eyes-open block-averaged resting-state NIRS was conducted at the central site Cz by inserting 1 source (760/850nm LED) through the $8 \mathrm{~cm}^{2}$ sponge (rubber core electrode) and with 4 symmetrically-placed detectors at $2.5 \mathrm{~cm}$ source-detector separation. NIRS was recorded at $22.5 \mathrm{~Hz}$ using a spatially resolved spectroscopy technique [24] and then pre-processed using HomER functions [25].

Also, eyes-open resting state EEG (StarStim, Neuroelectrics, Spain) was recorded at $500 \mathrm{~Hz}$ from the nearby electrodes F3, F4, P3, P4 (international 10-20 system), which were interpolated with spherical splines to estimate the EEG at $\mathrm{Cz}$ (virtual electrode) using EEGLAB 'eeg_interp()' function [26]. The EEG at the central site $\mathrm{Cz}$ (virtual electrode) before and immediately after anodal tDCS at $\mathrm{Cz}$ was pre-processed using EEGLAB functions [26]. 


\section{EEG-NIRS based assessment of tDCS effects}

Prior work has shown that tDCS induces changes in neuronal membrane potentials in a polarity-dependent manner and induces synaptically driven after-effects after a sufficient duration [27]. Basal and apical dendrites can be concomitantly polarized in opposite directions [7]. Consequently stimulation results in local excitatory (or inhibitory) postsynaptic current, which then results in an excitatory (or inhibitory) postsynaptic potential at the cell body causing respective membrane potential alterations. The synaptic transmembrane currents are a major contributor of the extracellular signal that can be measured with current source density (CSD) analysis of the extracellular field potentials, viz., local field potentials (LFP) [28]. Here, EEG monitored the extracellular field potentials which can be used to estimate the EEG surface laplacian [29] - the magnitude of the radial (transcranial) current flow leaving (sinks) and entering (sources) the scalp. These reference-free current source density (CSD) waveforms yield measures that closely represent underlying neuronal current generators [30], and therefore CSD waveforms at the site of stimulation, $\mathrm{Cz}$, should represent underlying neuronal current generators. Prior works show a strong coupling between LFP and regional vascular responses even in the absence of spikes (i.e., sub-threshold depolarization) [31]. Moreover, our prior work showed an increase of fractional power in the Theta band $(4-8 \mathrm{~Hz})$ and decrease around "individual alpha frequency" in the Alpha band (8-13Hz) following anodal tDCS [5]. In this study, NIRS complemented the electrophysiological measures with measurements of the changes in (cortical) tissue oxy-( $\mathrm{HbO} 2)$, and deoxy-( $\mathrm{Hb})$ hemoglobin concentration roughly underlying

$\mathrm{Cz}$ location. Total hemoglobin concentration, Hbt, is considered as a good indicator of variations in regional cerebral blood volume [32] which was derived as the sum of $\mathrm{HbO2}$ and $H b$ concentrations. 


\section{Hilbert-Huang Transform-based assessment of neurovascular coupling}

After pre-processing EEG in EEGLAB [26], Empirical Mode Decomposition (EMD) into a set of intrinsic mode functions $\left(\mathrm{IMF}_{\mathrm{EEG}}\right)$ was performed using the Hilbert-Huang Transform (HHT) [33]. Also, NIRS-measured changes in (cortical) tissue oxy-( $\mathrm{HbO2})$, and deoxy-( $\mathrm{Hb}$ ) haemoglobin concentration in the volume roughly underlying the $\mathrm{Cz}$ location, which was preprocessed in HomER [25]. After pre-processing, Empirical Mode Decomposition (EMD) into a set of intrinsic mode functions $\left(\mathrm{IMF}_{\mathrm{NIRS}}\right)$ was performed on the $\mathrm{HbO2}$ time-series. Resting-state neurovascular coupling from EEG-NIRS recordings was assessed as follows.

1. Empirical Mode Decomposition (EMD) of non-stationary electrophysiological and hemodynamic resting-state time-series into a set of intrinsic mode functions (IMFs) using the Hilbert-Huang Transform (HHT) [33]. Generally, the first IMF contains the highest frequency components and the oscillatory frequencies decrease with increasing IMF index. The IMFs for EEG are denoted as $\mathrm{IMF}_{\mathrm{EEG}}$ and IMFs for $\mathrm{HbO2}$ are denoted as IMF $\mathrm{NIRS}_{\text {. }}$

2. Then, the cross correlation function between the IMF $\mathrm{NIRS}_{\text {and }}$ ag-transformed mean-power time-series (non-overlapping 2 seconds moving window) of $\mathrm{IMF}_{\mathrm{EEG}}$ was obtained using MATLAB toolbox[34]. The component of $\mathrm{IMF}_{\mathrm{NIRS}}$ and $\mathrm{IMF}_{\mathrm{EEG}}$ that optimally represented the non-stationary effects of anodal tDCS was used for the assessment of neurovascular coupling.

3. For the assessment of neurovascular coupling (NVC) during anodal tDCS from the nonstationary $\mathrm{IMF}_{\mathrm{NIRS}}$ and $\mathrm{IMF}_{\mathrm{EEG}}$ components, the Hilbert transform of the $\mathrm{IMF}_{\mathrm{NIRS}}$ and logtransformed mean-power time-series of $\mathrm{IMF}_{\mathrm{EEG}}\left(\mathrm{PIMF}_{\mathrm{EEG}}\right)$ was first performed, 


$$
\begin{aligned}
& H_{E E G, i}(t)=\frac{1}{\pi} P \int_{-\infty}^{\infty} \frac{P I M F_{E E G, i}(\tau)}{t-\tau} d \tau \\
& H_{N I R S, i}(t)=\frac{1}{\pi} P \int_{-\infty}^{\infty} \frac{I M F_{N I R S, i}(\tau)}{t-\tau} d \tau
\end{aligned}
$$

where $P$ is the Cauchy principal value. Then, the analytic signals were defined as,

$$
\begin{aligned}
& Z_{E E G, i}(t)=P I M F_{E E G, i}(t)+i H_{E E G, i}(t) \\
& Z_{N I R S, i}(t)=I M F_{N I R S, i}(t)+i H_{N I R S, i}(t)
\end{aligned}
$$

The instantaneous amplitudes for the analytic signals were determined as,

$$
\begin{aligned}
& A_{E E G, i}(t)=\left[\operatorname{PIMF}_{E E G, i}^{2}(t)+H_{E E G, i}^{2}(t)\right]^{1 / 2} \\
& A_{N I R S, i}(t)=\left[I M F_{N I R S, i}^{2}(t)+H_{N I R S, i}^{2}(t)\right]^{1 / 2}
\end{aligned}
$$

The instantaneous phases for the analytic signals were determined as,

$$
\begin{aligned}
& \theta_{E E G, i}(t)=\arctan \left(\frac{H_{E E G, i}(t)}{P I M F_{E E G, i}(t)}\right) \\
& \theta_{N I R S, i}(t)=\arctan \left(\frac{H_{N I R S, i}(t)}{I M F_{N I R S, i}(t)}\right)
\end{aligned}
$$

The instantaneous frequency for the analytic signals was determined as,

$$
\begin{aligned}
& f_{E E G, i}(t)=\frac{1}{2 \pi} \frac{d \theta_{E E G, i}(t)}{d t} \\
& f_{N I R S, i}(t)=\frac{1}{2 \pi} \frac{d \theta_{N I R S, i}(t)}{d t}
\end{aligned}
$$

To compute cross-spectrum and coherence during anodal tDCS for the assessment of NVC, the component of $\mathrm{IMF}_{\mathrm{NIRS}}$ and $\mathrm{IMF}_{\mathrm{EEG}}$ that best represented the non-stationary effects of anodal tDCS were selected (say, $\operatorname{IMF}_{\text {NIRS,j}}(t)$ and $P I M F_{E E G, k}(t)$ ) for further analysis. 
4. The cross-spectrum and coherence between $P I M F_{E E G, k}(t)$ and $I M F_{N I R S, j}(t)$ during anodal tDCS were calculated based on their instantaneous amplitude and phase. Here, a moving window method was followed where the average instantaneous frequency was first computed. Then, the cross-spectrum at time instant, $t$, was computed for the frequency, $f_{p}$, from $m^{\text {th }}$ and $n^{\text {th }}$ observation windows (i.e., $P I M F_{E E G, k, m}(t)$ and $I M F_{N I R S, j, n}(t)$ ) which have average instantaneous frequency closest to $f_{p}$, i.e.,

$$
\begin{aligned}
& C_{f_{p}}\left(P I M F_{E E G, k}, I M F_{N I R S, j}\right)=A_{E E G, k, m}(t) A_{N I R S, j, n}(t) e^{i\left[\theta_{E E G, k, m}(t)-\theta_{N I R S, j, n}(t)\right]} \\
& C_{f_{p}}\left(I M F_{N I R S, j}, P I M F_{E E G, k}\right)=A_{N I R S, j, n}(t) A_{E E G, k, m}(t) e^{i\left[\theta_{N I R S, j, n}(t)-\theta_{E E G, k, m}(t)\right]}
\end{aligned}
$$

Also, the coherence was computed as,

$$
\begin{aligned}
\operatorname{Coh}_{E E G \rightarrow N I R S, f_{p}} & =\frac{\left\langle C_{f_{p}}\left(P I M F_{E E G, k}, I M F_{N I R S, j}\right)^{2}\right\rangle}{\left\langle\left[A_{E E G, k, m}(t) e^{i \theta_{E E G, k, m}(t)}\right]^{2}\right\rangle\left\langle\left[A_{N I R S, j, n}(t) e^{i \theta_{N I R S, j, n}(t)}\right]^{2}\right\rangle} \\
\operatorname{Coh}_{N I R S \rightarrow E E G, f_{p}} & =\frac{\left\langle C_{f_{p}}\left(I M F_{N I R S, j}, P I M F_{E E G, k}\right)^{2}\right\rangle}{\left\langle\left[A_{N I R S, j, n}(t) e^{i \theta_{N I R S, j, n}(t)}\right]^{2}\right\rangle\left\langle\left[A_{E E G, k, m}(t) e^{i \theta_{E E G, k, m}(t)}\right]^{2}\right\rangle}
\end{aligned}
$$

where \langle\rangle denotes averaging over multiple paired-windows for the given frequency, $f_{p}$. Significant positive values point to a causal relation [35].

5. The mean neurovascular coupling (NVC) for a given frequency range, $\Delta f_{p}$,.was defined as,

$$
\begin{aligned}
& N V C_{E E G \rightarrow N I R S}\left(\Delta f_{p}\right)=\left\langle\operatorname{Coh}_{E E G \rightarrow N I R S, f_{p}}\right\rangle \\
& N V C_{N I R S \rightarrow E E G}\left(\Delta f_{p}\right)=\left\langle\operatorname{Coh}_{N I R S \rightarrow E E G, f_{p}}\right\rangle
\end{aligned}
$$


where \langle\rangle denotes averaging over a given frequency range, $\Delta f_{p}$, over all the trials.

All computations were performed with custom-code developed in Matlab R2011a (The Mathworks Inc, USA).

\section{RESULTS}

The illustrative results from Case-1 are shown in Figure 2, where the top-panel of Figure 2a shows the regional change in tissue oxy- ( $\mathrm{HbO2}$ ), deoxy- ( $\mathrm{Hb}$ ), and total ( $\mathrm{Hbt}$ ) haemoglobin concentration from $0-60 \mathrm{sec}$ due to application of $0.526 \mathrm{~A} / \mathrm{m}^{2}$ anodal tDCS from $0-30 \mathrm{sec}$.

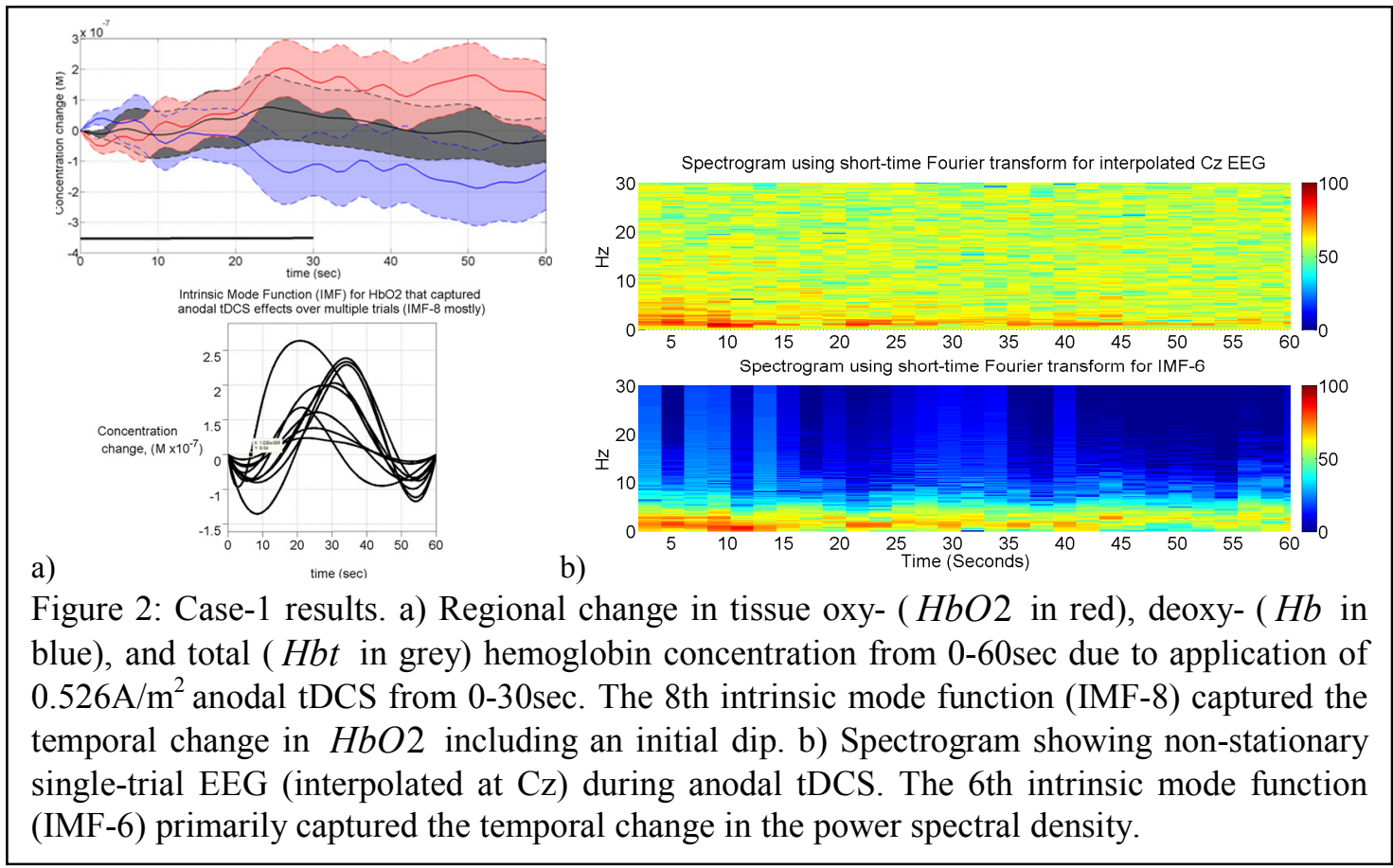

The 8th intrinsic mode function (IMF-8) mostly captured the temporal change in $\mathrm{HbO} 2$ over all trials $(\mathrm{N}=15)$ that represented non-stationary effects of anodal tDCS including the initial dip, as shown in the bottom panel of Figure 2a. The duration of this initial dip was computed from the IMF-8 time-series for each trial for all subjects. The initial dip-duration for Case-1 
$(12.13 \pm 3.81 \mathrm{sec})$ was much longer than those of the other three cases $(6.04 \pm 3.82 \mathrm{sec})$. The corresponding EEG spectrogram for Case-1 using short-time Fourier transform for the interpolated EEG estimate at $\mathrm{Cz}$ (virtual electrode) is shown in the top panel of Figure 2b. Here, the 6th intrinsic mode function (IMF-6) mostly captured the non-stationary effects of anodal tDCS on the power spectral density (PSD) within the $0.5 \mathrm{~Hz}-11.25 \mathrm{~Hz}$ frequency band, which is shown in the bottom panel of Figure $2 \mathrm{~b}$. Therefore, IMF-8 for $\mathrm{HbO} 2$ and IMF-6 for interpolated EEG at $\mathrm{Cz}$ (virtual electrode) were selected for the assessment of neurovascular coupling (NVC) during anodal tDCS. We investigated NVC during tDCS, assuming that anodal tDCS facilitated cortical neural activity, resulting in glutamate release into the synapse, and consequently facilitating astrocyte-mediated vasodilation, which was assessed with $N V C_{E E G \rightarrow N I R S}(0.5 \mathrm{~Hz}-11.25 \mathrm{~Hz})$.
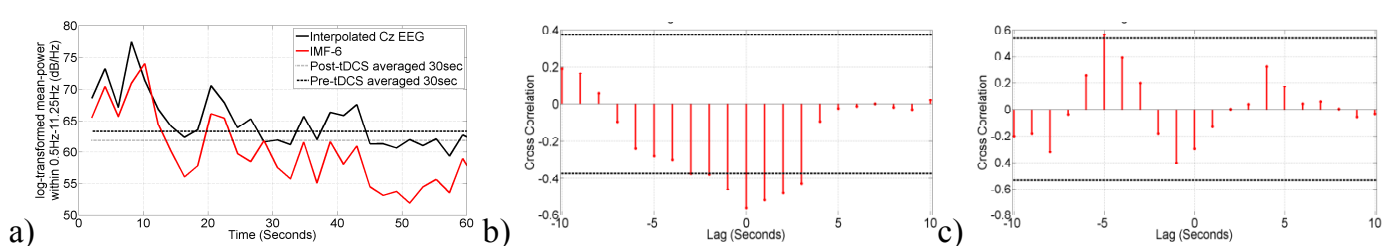

Figure 3: Case-1 results. a) the 6th Intrinsic Mode Function (IMF-6) of the interpolated EEG estimate at $\mathrm{Cz}$ (virtual electrode) captured the temporal change in the log-transformed meanpower (within $0.5 \mathrm{~Hz}-11.25 \mathrm{~Hz}$ ) of the EEG. b) Cross-correlation function during anodal tDCS between log-transformed mean-power time-course of $I M F_{E E G}$ and $I M F_{H b O 2}$ time-series. c) Cross-correlation function during post-tDCS resting-state (i.e., no task/no tDCS) between log-transformed mean-power time-course of $I M F_{E E G}$ and $I M F_{H b O 2}$ time-series. In b) and c), dashed lines show 95 percent confidence interval for the assumption that the two time series are completely uncorrelated.

Figure 3a compares the log-transformed mean-power of IMF-6 with the log-transformed mean-power of EEG (interpolated at $\mathrm{Cz}$ ) within $0.5 \mathrm{~Hz}-11.25 \mathrm{~Hz}$. It was found that $\log$ transformed mean-power time-series of IMF-6 captured the log-transformed mean-power timecourse of the EEG (interpolated at $\mathrm{Cz}$ ) within $0.5 \mathrm{~Hz}-11.25 \mathrm{~Hz}$, showing non-stationary effects of 
anodal tDCS (see Figure $2 b$ ). Figure $3 b$ shows the cross-correlation function between the logtransformed mean-power time-series of IMF-6 (Figure 3a) and the IMF-8 of $\mathrm{HbO} 2$ (Figure 2a). Here, a negative cross correlation of $\sim 0.6$ with a zero lag (95 percent confidence interval) was found for this trial during anodal tDCS. Figure $3 \mathrm{c}$ shows the cross-correlation function between the log-transformed mean-power time-series of IMF-6 (Figure 3a) and the IMF-8 of $\mathrm{HbO2}$ (Figure 2a) during post-tDCS resting-state (i.e., no task and no tDCS), which was found to be positive $\sim 0.6$ with a lead of $5 \sec (95$ percent confidence interval). The results including the $N V C_{E E G \rightarrow N I R S}(0.5 \mathrm{~Hz}-11.25 \mathrm{~Hz})$ during anodal tDCS from all the trials $(\mathrm{N}=15)$ for all four cases are listed in Table 2.

Table 2: Summary of the results from the case series.

\begin{tabular}{|c|c|c|c|c|c|}
\hline Case & \multicolumn{3}{|c|}{$\begin{array}{c}\text { During tDCS } \\
\text { (mean } \pm \text { 1 std. dev.) }\end{array}$} & \multicolumn{2}{c|}{$\begin{array}{c}\text { Post-tDCS } \\
\text { (mean } \pm \text { 1 std. dev.) }\end{array}$} \\
\hline $\begin{array}{c}\text { Trials } \\
(\mathbf{N}=\mathbf{1 5})\end{array}$ & $\begin{array}{c}\text { Correlation } \\
\text { Coefficient }\end{array}$ & $\begin{array}{l}N V C_{E E G \rightarrow N I R S} \\
(0.5 \mathrm{~Hz}-11.25 \mathrm{~Hz})\end{array}$ & Lag (sec) & $\begin{array}{c}\text { Correlation } \\
\text { Coefficient }\end{array}$ & Lag (sec) \\
\hline 1 & $-0.56 \pm 0.21$ & 0.93 & $0.93 \pm 1.26$ & $0.57 \pm 0.16$ & $-5.19 \pm 1.42$ \\
\hline 2 & $-0.64 \pm 0.15$ & 0.81 & $3.28 \pm 1.38$ & $0.62 \pm 0.19$ & $-4.75 \pm 1.57$ \\
\hline 3 & $-0.58 \pm 0.13$ & 0.79 & $2.75 \pm 1.96$ & $0.59 \pm 0.18$ & $-6.09 \pm 1.29$ \\
\hline 4 & $-0.55 \pm 0.19$ & 0.78 & $3.56 \pm 1.19$ & $0.58 \pm 0.12$ & $-6.18 \pm 1.17$ \\
\hline
\end{tabular}

V. DISCUSSION

The results of this case series shows that anodal tDCS induces a local neurovascular response which may be used for assessing regional neurovascular coupling (NVC) functionality. It was postulated that $\mathrm{tDCS}$ leads to rapid dynamic variations of the brain cell microenvironment [36] that perturbs hemodynamic and electrophysiological responses. Indeed, we found immediate regional changes in tissue oxy- $(\mathrm{HbO} 2)$ and deoxy- $(\mathrm{Hb})$ haemoglobin concentration from 0 $60 \mathrm{sec}$ due to application of $0.526 \mathrm{~A} / \mathrm{m}^{2}$ anodal tDCS from $0-30 \mathrm{sec}$ (Figure 2a). Also, non- 
stationary effects of anodal tDCS on EEG (interpolated at $\mathrm{Cz}$ ) were identified from the spectrogram, as shown in Figure 2b. Here, the log-transformed mean-power within $0.5 \mathrm{~Hz}$ $11.25 \mathrm{~Hz}$ frequency band was much higher at the beginning $(0-10 \mathrm{sec})$ of anodal tDCS when compared to post-tDCS $(>30 \mathrm{sec})$ values in Case-1 (Figure 3a). This non-stationary logtransformed mean-power within the $0.5 \mathrm{~Hz}-11.25 \mathrm{~Hz}$ frequency band was best captured by the 6 th intrinsic mode function, IMF-6 (Figure 3a). Also, it was found that the 8th intrinsic mode function (IMF-8) mostly captured the non-stationary effects of anodal tDCS on $\mathrm{HbO2}$ timeseries including the initial dip $(12.13 \pm 3.81 \mathrm{sec}$ in Case-1), as shown in the bottom panel of Figure 2a. Therefore, IMF-8 for $\mathrm{HbO} 2$ and IMF-6 for interpolated EEG at $\mathrm{Cz}$ (virtual electrode) were selected for the assessment of neurovascular coupling (NVC) during anodal tDCS, which is summarized in Table 2. During anodal tDCS, the negative cross-correlation coefficient was found to be comparable across cases and log-transformed mean-power of IMF- 6 for interpolated EEG at $\mathrm{Cz}$ (virtual electrode) lagged IMF-8 for $\mathrm{HbO2}$. However, the lag for Case-1 $(0.93 \pm 1.26 \mathrm{sec})$ differed from the other cases. Case-1, a 31 years old left hemiplegic male subject, also complained of headache with throbbing pain following 15 minutes of anodal tDCS in another study. For post-tDCS, the cross-correlation coefficient was positive with a negative lag (i.e., IMF-8 for $\mathrm{HbO} 2$ lagged log-transformed mean-power of IMF-6 for EEG), which were comparable across subjects.

We found log-transformed mean-power within $0.5 \mathrm{~Hz}-11.25 \mathrm{~Hz}$ frequency band much higher at the beginning $(\sim 0-10 \mathrm{sec})$ of anodal tDCS when compared to post-tDCS $(>30 \mathrm{sec})$ values in Case-1 (Figure 3a). Since moderate rises in the extracellular potassium concentration has been shown to depress high-frequency synaptic transmission [37] which may lead to an increase in power in the lower $(0.5 \mathrm{~Hz}-11.25 \mathrm{~Hz})$ frequency band so we postulate that anodal tDCS may 
cause a rapid increase in the extracellular potassium concentration due to outwardly directed potassium currents. Here, diffusing extracellular potassium ions can act as mediators of vasodilation [14] as well as can act on the neuronal compartments, including GABAergic and glutamatergic synapses, which might contribute to the aftereffects of anodal tDCS [15]. Also, an immediate response towards the recovery of homeostasis can be triggered by the extracellular potassium (and, may be calcium [10]) ion movement during perturbation with anodal tDCS. This recovery (and maintenance) of homeostasis in the cortex [18] during perturbation by anodal tDCS can be fuelled by glucose (and lactate) uptake by astrocytes and neurons from the interstitium. Subsequent increase in the cerebral metabolic rate of oxygen due to aerobic energy metabolism might have led to a negative cross-correlation between the $\mathrm{HbO} 2$ time-series and log-transformed mean-power time-course of EEG primarily within $0.5 \mathrm{~Hz}-11.25 \mathrm{~Hz}$ frequency band (Table 2). Here, we postulate that the immediate need to fuel recovery of homeostasis was caused by astrocytes via the lactate shuttle [16] while blood glucose supply has a longer delay [38]. Although physiologically stimulated increases in glucose and lactate in the brain have been shown to be mediated by different mechanisms [39], the functional role of potassium dynamics (in addition to calcium wave dynamics [10]) as a homeostatic feedback mechanism should not be discounted since an augmentation of extracellular potassium concentration can cause depolarization of astrocytes, where the glycogen in the brain is localized almost exclusively, leading to glycogen degradation to lactate and a portion transferred to axons as fuel [40]. Therefore, it was hypothesized for Case-1 that the initial $(\sim 0-10 \mathrm{sec})$ dip in $\mathrm{HbO2}$ at the beginning of anodal tDCS (Figure 2a) and corresponding increase in the log-transformed meanpower within the $0.5 \mathrm{~Hz}-11.25 \mathrm{~Hz}$ frequency band (Figure $2 \mathrm{~b}$ ) were related to the recovery of homeostasis. Interestingly, these alterations lasted longer (12.13 $\pm 3.81 \mathrm{sec}$ dip-duration) for Case- 
1 than other three cases $(6.04 \pm 3.82 \mathrm{sec})$, and only Case- 1 complained about headache after tDCS [19]. Here, it is known that cerebral blood flow is increased in the brain regions with neural activity via metabolic coupling mechanisms whereas the autoregulation mechanisms ensure that the blood flow is maintained during changes in the perfusion pressure. Therefore, this dipduration in $\mathrm{HbO2}$ at the beginning of anodal tDCS that was captured using Intrinsic Mode Function needs to be carefully investigated in a larger ischemic stroke patient group, for example, with small and large vessel disease to test the hypothesis that patients with small vessel disease are more impaired on neurovascular coupling associated with microcirculatory function than stroke patient with large intracranial artery stenosis.

Towards this overarching goal, it is also our objective to develop low-cost point-of-caretesting device based on this EEG-NIRS based assessment of neurovascular coupling during anodal transcranial direct current stimulation. A custom-made continuous wave (CW) NIRS system based on an off-the-shelf data acquisition (DAQ) device to interface between signal and computer may provide the user with a robust, fast and flexible solution [41]. A multifunction offthe-shelf DAQ device (National Instruments Corporation, USA) can be used to provide basic physical input/output channels to drive the light sources and/or acquire optical signals. If the timing accuracy of DAQ is critical, e.g. for cross spectral analysis [42], then a real-time off-theshelf DAQ system, such as CompactRIO or PXI (National Instruments Corporation, USA), is required. Usually, the analog output channels are limited in off-the-shelf DAQ devices (e.g., only 2 analog outputs in PCIe-6351 from National Instruments Corporation, USA) and therefore time division multiplexing technique can be used to illuminate light sources (light emitting diodes or laser diodes). For example, the NIR light sources can be amplitude modulated at different carrier frequencies with a gap (viz. 2 to $4 \mathrm{KHz}$ in steps of $200 \mathrm{~Hz}$ ) using a driving 
circuit that includes a multiplexer and driver for the laser diode (LD) or light emitting diode (LED) to emit NIR light in consecutive time slots. LED, which emit incoherent and uncollimated light, is preferred since it allows the emission of more NIR photons into the tissue than by LD with the same maximum permissible exposure [41]. Here, dual-wavelength LEDs can be used in a high density whole head system. Moreover, an individual programmable current source for each LED can be used so that the emitted light can be automatically adapted to variable individual acquisition conditions (hair, skin color, different tissue types such as muscle, brain etc as well as a possible effect of EEG gel on the optical properties of the light path) [43]. Then, the backscattered NIR light can be collected (light collected is usually 7 to 9 orders smaller in magnitude than that emitted at the source [44]) and converted into electrical signal by photodiodes. Due to low signal to noise ratio (SNR) of the output signal from the photodiodes, the signal can be band-pass filtered and amplified to increase the SNR. A programmable gain amplifier (e.g. PGA204, Texas Instruments Inc., USA) can be used to adapt the gain depending on source-detector distance, scalp thickness, and hair color and density [45] to match the voltage range of the analog input channel of the DAQ device. Moreover, integration of EEG to CW NIRS device should not increase the cost significantly since low-noise, 8-Channel, 24-Bit Analog Front-End for EEG measurements are available for \$200 (ADS1299EEG-FE, Texas Instruments, USA). Furthermore, a low-cost tDCS hardware can be based on a current regulator that sets and stabilizes the current at a fixed level. The current can be controlled using a three terminal adjustable current source (LM334Z, Texas Instruments, USA) - featuring 10,000:1 range in operating current, excellent current regulation and a wide dynamic voltage range of $1 \mathrm{~V}$ to $40 \mathrm{~V}$ - where the current level was set with one external resistor, i.e., an analog potentiometer (Sood et al. 2014). Using the EEG-NIRS signals evoked under tDCS, periodogram and 
autoregressive spectral analysis approaches can be applied for feature extraction [47][48][49] towards knowledge-based diagnosis using fuzzy modeling and a knowledge based approach to classifier design [50] in a point-of-care device [51][52]. This custom-made EEG-NIRS/tDCS system based on an off-the-shelf DAQ device is currently under development and the design documents can be availed at https://team.inria.fr/nphys4nrehab/hardware/

\section{ACKNOWLEDGMENTS}

Research conducted within the context of the Franco-German PHC-PROCOPE 2014 funding. A. Jacob would like to thank German Academic Exchange Service (DAAD) for supporting her internship in Germany. The help received from the Neuro Rehab Services LLP, India in conducting the clinical study is gratefully acknowledged. This project was further supported by the German Ministry for Education and Research (BMBF, Project EYE-TSS 03IPT605E).

\section{REFERENCES}

[1] Nitsche MA, Paulus W. Excitability changes induced in the human motor cortex by weak transcranial direct current stimulation. J Physiol. 2000 Sep 15;527 Pt 3:633-9.

[2] Nunez PL, Srinivasan R. Electric Fields of the Brain: The Neurophysics of EEG. Oxford University Press; 2006. 629 p.

[3] Girouard H, Iadecola C. Neurovascular coupling in the normal brain and in hypertension, stroke, and Alzheimer disease. J Appl Physiol Bethesda Md 1985. 2006 Jan;100(1):328_ 35 .

[4] Siesler HW, Ozaki Y, Kawata S, Heise HM. Near-Infrared Spectroscopy: Principles, Instruments, Applications. John Wiley \& Sons; 2008. 365 p.

[5] A. Dutta, M.A. Nitsche. A neural mass model for simulating modulation of cortical activity with transcranial direct current stimulation. In proceeding of: 2013 6th International IEEE/EMBS Conference on Neural Engineering (NER).

[6] David O, Friston KJ. A neural mass model for MEG/EEG: coupling and neuronal dynamics. NeuroImage. 2003 Nov;20(3):1743-55. 
[7] Rahman A, Reato D, Arlotti M, Gasca F, Datta A, Parra LC, et al. Cellular effects of acute direct current stimulation: somatic and synaptic terminal effects. J Physiol. 2013 May 15;591(Pt 10):2563-78.

[8] Radman T, Ramos RL, Brumberg JC, Bikson M. Role of cortical cell type and morphology in subthreshold and suprathreshold uniform electric field stimulation in vitro. Brain Stimulat. 2009 Oct;2(4):215-228, 228.e1-3.

[9] Holthoff K, Witte OW. Directed spatial potassium redistribution in rat neocortex. Glia. 2000 Feb 1;29(3):288-92.

[10] 10. Fricke K, Seeber AA, Thirugnanasambandam N, Paulus W, Nitsche MA, Rothwell JC (2011) Time course of the induction of homeostatic plasticity generated by repeated transcranial direct current stimulation of the human motor cortex. J Neurophysiol 105:1141-1149. doi: 10.1152/jn.00608.2009

[11] 11. Islam N, Aftabuddin M, Moriwaki A, Hattori Y, Hori Y (1995) Increase in the calcium level following anodal polarization in the rat brain. Brain Res 684:206-208.

[12] 12. Nitsche MA, Fricke K, Henschke U, Schlitterlau A, Liebetanz D, Lang N, Henning S, Tergau F, Paulus W (2003) Pharmacological modulation of cortical excitability shifts induced by transcranial direct current stimulation in humans. J Physiol 553:293-301. doi: 10.1113/jphysiol.2003.049916

[13] Halnes G, Ostby I, Pettersen KH, Omholt SW, Einevoll GT. Electrodiffusive Model for Astrocytic and Neuronal Ion Concentration Dynamics. PLoS Comput Biol [Internet]. 2013 Dec [cited 2014 May 11];9(12).

[14]Eckman DM, Nelson MT. Potassium Ions as Vasodilators: Role of Inward Rectifier Potassium Channels. Circ Res. 2001 Feb 2;88(2):132-3.

[15] Stagg CJ, Nitsche MA. Physiological basis of transcranial direct current stimulation. Neurosci Rev J Bringing Neurobiol Neurol Psychiatry. 2011 Feb;17(1):37-53.

[16] Pellerin L, Magistretti PJ. Glutamate uptake into astrocytes stimulates aerobic glycolysis: a mechanism coupling neuronal activity to glucose utilization. Proc Natl Acad Sci U S A. 1994 Oct 25;91(22):10625-9.

[17] DeFazio RA, Keros S, Quick MW, Hablitz JJ. Potassium-coupled chloride cotransport controls intracellular chloride in rat neocortical pyramidal neurons. J Neurosci Off J Soc Neurosci. 2000 Nov 1;20(21):8069-76.

[18] Hübel N, Schöll E, Dahlem MA. Bistable dynamics underlying excitability of ion homeostasis in neuron models. PLoS Comput Biol. 2014 May;10(5):e1003551.

[19] A. Dutta, S. Roy Chowdhury, A. Das, "A Novel Method For Capturing Cerebrovascular Reactivity Using Near-infrared Spectroscopy During Transcranial Direct Current 
Stimulation: A Stroke Case Series," 30th International Congress of Clinical Neurophysiology 2014.

[20] Bozzo L, Puyal J, Chatton J-Y. Lactate Modulates the Activity of Primary Cortical Neurons through a Receptor-Mediated Pathway. PLoS ONE. 2013 Aug 12;8(8):e71721.

[21] Nikulin VV, Fedele T, Mehnert J, Lipp A, Noack C, Steinbrink J, et al. Monochromatic Ultra-Slow $(\sim 0.1 \mathrm{~Hz})$ Oscillations in the human electroencephalogram and their relation to hemodynamics. NeuroImage. 2014 Apr 13;

[22] A. Dutta, "EEG-NIRS based low-cost screening and monitoring of cerebral microvessels functionality," International Stroke Conference 2014, San Diego, 2014.

[23] Barbour RL, Graber HL, Xu Y, Pei Y, Schmitz CH, Pfeil DS, et al. A programmable laboratory testbed in support of evaluation of functional brain activation and connectivity. IEEE Trans Neural Syst Rehabil Eng Publ IEEE Eng Med Biol Soc. 2012 Mar;20(2):170-83.

[24] Choi J, Wolf M, Toronov V, Wolf U, Polzonetti C, Hueber D, et al. Noninvasive determination of the optical properties of adult brain: near-infrared spectroscopy approach. J Biomed Opt. 2004 Feb;9(1):221-9.

[25] Huppert TJ, Diamond SG, Franceschini MA, Boas DA. HomER: a review of time-series analysis methods for near-infrared spectroscopy of the brain. Appl Opt. 2009 Apr 1;48(10):D280-298.

[26] Delorme A, Makeig S. EEGLAB: an open source toolbox for analysis of single-trial EEG dynamics including independent component analysis. J Neurosci Methods. 2004 Mar $15 ; 134(1): 9-21$.

[27] Molaee-Ardekani B, Márquez-Ruiz J, Merlet I, Leal-Campanario R, Gruart A, SánchezCampusano R, et al. Effects of transcranial Direct Current Stimulation (tDCS) on cortical activity: A computational modeling study. Brain Stimulat. 2013 Jan;6(1):25-39.

[28] Buzsáki G, Anastassiou CA, Koch C. The origin of extracellular fields and currents--EEG, ECoG, LFP and spikes. Nat Rev Neurosci. 2012 Jun;13(6):407-20.

[29] Carvalhaes CG, Suppes P. A spline framework for estimating the EEG surface laplacian using the Euclidean metric. Neural Comput. 2011 Nov;23(11):2974-3000.

[30] Perrin F, Pernier J, Bertrand O, Echallier JF. Spherical splines for scalp potential and current density mapping. Electroencephalogr Clin Neurophysiol. 1989 Feb;72(2):184-7.

[31] Viswanathan A, Freeman RD. Neurometabolic coupling in cerebral cortex reflects synaptic more than spiking activity. Nat Neurosci. 2007 Oct;10(10):1308-12. 
[32] Villringer A, Chance B. Non-invasive optical spectroscopy and imaging of human brain function. Trends Neurosci. 1997 Oct;20(10):435-42.

[33] Huang NE, Shen Z, Long SR, Wu MC, Shih HH, Zheng Q, et al. The empirical mode decomposition and the Hilbert spectrum for nonlinear and non-stationary time series analysis. Proc R Soc Lond Ser Math Phys Eng Sci. 1998 Mar 8;454(1971):903-95.

[34]Zhou D, Thompson WK, Siegle G. MATLAB toolbox for functional connectivity. NeuroImage. 2009 Oct 1;47(4):1590-607.

[35] Dhamala M, Rangarajan G, Ding M. Analyzing information flow in brain networks with nonparametric Granger causality. NeuroImage. 2008 Jun;41(2):354-62.

[36] Dietzel I, Heinemann U. Dynamic variations of the brain cell microenvironment in relation to neuronal hyperactivity. Ann N Y Acad Sci. 1986;481:72-86.

[37] Meeks JP, Mennerick S. Selective effects of potassium elevations on glutamate signaling and action potential conduction in hippocampus. J Neurosci Off J Soc Neurosci. 2004 Jan 7;24(1):197-206.

[38] Gruetter R, Novotny EJ, Boulware SD, Rothman DL, Shulman RG. 1H NMR Studies of Glucose Transport in the Human Brain. J Cereb Blood Flow Metab. 1996 May;16(3):427-38.

[39] Fray AE, Forsyth RJ, Boutelle MG, Fillenz M. The mechanisms controlling physiologically stimulated changes in rat brain glucose and lactate: a microdialysis study. J Physiol. 1996 Oct 1;496 ( Pt 1):49-57.

[40] Brown AM, Ransom BR (2007) Astrocyte glycogen and brain energy metabolism. Glia 55:1263-1271. doi: 10.1002/glia.20557

[41] 41. Soraghan C, Matthews F, Markham C, Pearlmutter BA, O’Neill R, Ward TE (2008) A 12-channel, real-time near-infrared spectroscopy instrument for brain-computer interface applications. Conf Proc Annu Int Conf IEEE Eng Med Biol Soc IEEE Eng Med Biol Soc Annu Conf 2008:5648-5651. doi: 10.1109/IEMBS.2008.4650495

[42] 42. Anirban Dutta MM (2014) Development of an EEG-fNIRS based online monitoring tool towards delivery of non-invasive brain stimulation.

[43] 43. Safaie J, Grebe R, Abrishami Moghaddam H, Wallois F (2013) Toward a fully integrated wireless wearable EEG-NIRS bimodal acquisition system. J Neural Eng 10:056001. doi: 10.1088/1741-2560/10/5/056001

[44] 44. Lareau E, Simard G, Lesage F, Nguyen D, Sawan M (2011) Near infrared spectrometer combined with multichannel EEG for functional brain imaging. 2011 5th Int. Symp. Med. Inf. Commun. Technol. ISMICT. pp 122-126 
[45] 45. Scholkmann F, Kleiser S, Metz AJ, Zimmermann R, Mata Pavia J, Wolf U, Wolf M (2014) A review on continuous wave functional near-infrared spectroscopy and imaging instrumentation and methodology. NeuroImage 85 Pt 1:6-27. doi: 10.1016/j.neuroimage.2013.05.004

[46] 46. Mehak Sood UJ (2014) Continuous wave functional near infra-red spectroscopy combined with transcranial direct current stimulation for assessment of cerebral vascular status in patients with ischemic stroke.

[47] 47. Akin M, Kiymik MK (2000) Application of periodogram and AR spectral analysis to EEG signals. J Med Syst 24:247-256.

[48] 48. Kiymik MK, Subasi A, Ozcalik HR (2004) Neural networks with periodogram and autoregressive spectral analysis methods in detection of epileptic seizure. J Med Syst 28:511-522.

[49] 49. Ubeyli ED, Cvetkovic D, Cosic I (2008) AR spectral analysis technique for human PPG, ECG and EEG signals. J Med Syst 32:201-206.

[50] 50. Moreno L, Sánchez JL, Mañas S, Piñeiro JD, Merino JJ, Sigut J, Aguilar RM, Estévez JI, Marichal R (2001) Tools for acquisition, processing and knowledge-based diagnostic of the electroencephalogram and visual evoked potentials. J Med Syst 25:177-194.

[51] 51. Das S, Roy Chowdhury S, Saha H (2012) Accuracy enhancement in a fuzzy expert decision making system through appropriate determination of membership functions and its application in a medical diagnostic decision making system. J Med Syst 36:16071620. doi: 10.1007/s10916-010-9623-8

[52] 52. Roy Chowdhury S, Roy A, Saha H (2011) ASIC design of a digital fuzzy system on chip for medical diagnostic applications. J Med Syst 35:221-235. doi: 10.1007/s10916-0099359-5 